\title{
The Mimas Ghost Revisited: An Analysis of the Electron Flux and Electron Microsignatures Observed in the Vicinity of Mimas at Saturn
}

\author{
D. L. Chenette \\ Space Sciences Laboratory, The Aerospace Corporation, Los Angeles, California 90009 \\ E. C. STONE
}

California Institute of Technology, Pasadena, California 91125

\begin{abstract}
An analysis of the electron absorption signature observed by the Cosmic Ray Sysiem un Vuyager 2 near the orbit of Mimas is presented. We find that these observations cannot be explained as the absorption signature of Mimas. Combining Pioneer 11 and Voyager 2 measurements of the electron flux at Mimas's orbit $(L=3.1$ ), we find an electron spectrum where most of the flux above $\sim 100 \mathrm{keV}$ is concentrated near 1 to $3 \mathrm{MeV}$. This spectral form is qualitatively consistent with the band-pass filter model of Van Allen et al. (1980b). The expected Mimas absorption signature is calculated from this spectrum neglecting radial diffusion. Since no Mimas absorption signature was observed in the inbound Voyager 2 data, a lower limit on the diffusion coefficient for $\mathrm{MeV}$ electrons at $L=3.1$ of $D>$ $10^{-8} R_{s}^{2} \mathrm{~s}^{-1}$ is obtained. With a diffusion coefficient this large, both the Voyager 2 and the Pioneer 11 small-scale electron absorption signature observations in Mimas's orbit are enigmatic. Thus we refer to the mechanism for producing these signatures as the Mimas ghost. A cloud of material in orbit with Mimas may account for the observed electron signature if the cloud is at least $1 \%$ opaque to electrons across a region extending over a few hundred kilometers.
\end{abstract}

\section{INTRODUCTION}

The satellites orbiting within the magnetospheres of Jupiter and Saturn have profound effects on the dynamics of these magnetospheres and the distributions of the trapped radiation. Some of these satellites are sources which contribute signifcant amounts of low-energy material to the magnetosphere (e.g., the Io torus [Broadfoot et al., 1979; Bagenal and Sullivan, 1981]), while others may be viewed as primarily as radiation sinks, absorbing energetic charged particles [Mead and Hess, 1973; Thomsen, 1979, and references therein]. As magnetospheric probes, these satellites have contributed greatly to our understanding of the structure, the dynamics, and the transport of the magnetospheric radiation. Conversely, the characteristic absorption signatures left in the radiation 'wake' of orbiting material has led to the confirmation or discovery of several new satellites and rings [Fillius et al., 1980; Simpson et al., 1980a, b; Van Allen et al., 1980a, b]. In this paper we exploit both of these aspects of charged particle/satellite interactions.

During their encounters with Saturn, both Pioneer 11 and Voyager 2 crossed the magnetic $L$ shell range occupied by Saturn's satellite Mimas. Energetic charged particle detectors onboard both of these spacecraft have measured transient decreases in the local electron flux which are similar to the characteristic signatures of charged particle absorption by material orbiting Saturn [Simpson et al., 1980b; Van Allen et al., 1980 ; Vogt et al., 1982; Carbary et al., this issue]. The interpretation of these features, however, has led to differing conclusions concerning the abundance of material sharing Mimas orbit. The resolution of these differences is important in order to assess the nature and the abundance of co-orbital material. Both Dione and Tethys have satellite companions [Smith et

Copyright 1983 by the American Geophysical Union.

Paper number $3 \mathrm{~A} 0617$. 0148-0227/83/003A-0617\$05.00 al., 1982], and these observations at Mimas may lead to a better understanding of the formation and the evolution of such multibody systems. Also, differences in the interpretation of these features have led to widely varying estimates of the radial diffusion coefficient for electrons near Mimas's orbit [Simpson et al., 1980b; Van Allen et al., 1980b]. The value of the radial diffusion coefficient is important for understanding the processes responsible for the formation and maintenance of Saturn's radiation belts.

In this paper we present an analysis of the energetic charged particle environment and the charged particle signatures observed in the vicinity of Mimas. In addition to summarizing the characteristics of the charged particle flux near Mimas, we derive limits on the radial diffusion coefficient for electrons in this region. Several candidate mechanisms are examined for producing the observed electron signature. Absorption by a cloud of material in orbit with Mimas appears to be a plausible explanation, and we derive limits on the characteristics of the additional material which may have been responsible for the absorption signature that we observed.

This analysis is based on data obtained from the Cosmic Ray System (CRS) on Voyager 2 [Stone et al., 1977]. During the Saturn encounter this instrument was operated in special modes to optimize its performance in the intense radiation regions of Saturn's inner magnetosphere [Schardt and McDonald, this issue]. Table 1 summarizes the characteristics of the CRS detectors which produced the data analyzed in this report. No corrections for nonlinearities between the incident flux intensity and the measured counting rate have been applied to these data. Such corrections become significant at measured rates greater than $2.4 \times 10^{4} \mathrm{~s}^{-1}$, which is greater than the maximum counting rate in Figure 1.

\section{Proton and Electron Profiles at Mimas}

Samples of the data obtained by the CRS instrument across the orbit of Mimas inbound and outbound are displayed in 
TABLE 1. Thresholds, Geometry Factors, and Radial Gradients at $L=3.1$ for CRS Electron Rates

\begin{tabular}{cccccc}
\hline $\begin{array}{c}\text { Detector } \\
\text { Name }\end{array}$ & $\begin{array}{c}\text { Electronic } \\
\text { Threshold, } \\
\mathbf{M e V}\end{array}$ & $\begin{array}{c}\text { Minimum } \\
\text { Shielding, } \\
\mathrm{g} \mathrm{cm}^{-2} ; \mathbf{A} 1\end{array}$ & $\begin{array}{c}\text { Geometry } \\
\text { Factor, } \\
\mathrm{cm}^{2} \mathbf{s r}\end{array}$ & $\begin{array}{c}\text { Incident Electron } \\
\text { Energy Threshold, } \\
\mathbf{M e V}\end{array}$ & $\begin{array}{c}\text { Counting Rate } \\
\text { Radial Gradient, } \\
\boldsymbol{R}_{\boldsymbol{s}}{ }^{-1}\end{array}$ \\
\hline B2 & 2.2 & 0.2 & 25. & 2.5 & 1.0 \\
D4 & 0.5 & 2.8 & 2.3 & 5. & 4.7 \\
C4 & 5. & 1. & 28. & 6.5 & 7.3 \\
C2 & 5. & 1.5 & 28. & 8. & $\approx 0$ \\
C3 & 5. & 2. & 11. & 9. & \\
\hline
\end{tabular}

Figure 1. The profiles of four different counting rate channels are plotted versus time (upper scale) and magnetic $L$ (lower scale). Throughout this paper, the $L$ shell coordinates of Voyager 2 have been calculated from the final spacecraft trajectory coordinates by assuming a magnetic dipole aligned with Saturn's axis of rotation and offset along Saturn's rotation axis by 0.04 Saturn radii $\left(R_{s}\right)$ north [Smith et al., 1980; Connerney et al., 1982; Chenette and Davis, 1982]. If the octopole moment term of the $Z_{s}$ magnetic field model [Connerney et al., 1982] were included in our calculation, the $L$ shell coordinates would change by less than $1 \%$. Exact $L$ values are not essential to the analysis that follows.

The counting rate of Figure 1 labeled BS4 is nominally the coincidence rate between detectors with nominal thresholds of $2.2 \mathrm{MeV}$ (detector B2) and $5 \mathrm{MeV}$ (detector $\mathrm{C4}$ ). In the region plotted in Figure 1, this rate is dominated by accidental coincidences between these two detectors. Therefore the BS4 rate is roughly proportional to the product of the two singledetector counting rates. Since the $>2.5-\mathrm{MeV}$ electron rate is from the B2 detector, the ratio of the BS4 to $>2.5-\mathrm{MeV}$ electron rate is roughly proportional to the flux of the $>6.5-\mathrm{MeV}$ electrons which can trigger the $\mathrm{C} 4$ detector.

In Figure 1, the proton and electron profiles are significantly different. The $>60-\mathrm{MeV}$ proton flux from the CRS instrument [Vogt et al., 1982; Schardt and McDonald, this issue] has a broad minimum from $L=3.02$ to 3.14 which is coincident with the semimajor axis of Mimas's orbit at 3.08

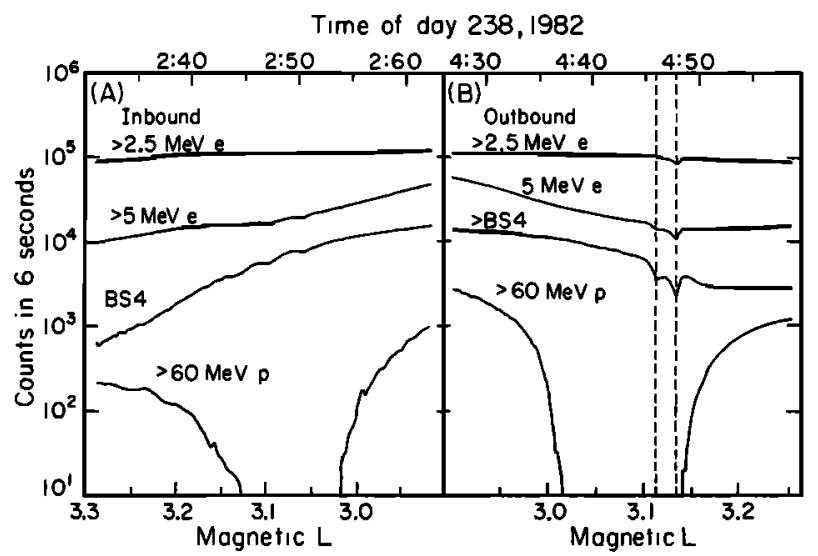

Fig. 1. Electron and proton counting rates from the CRS instrument on Voyager 2 as the spacecraft crossed the orbit of Mimas both (a) inbound and $(b)$ outbound. BS4 labels a rate which is responding to the product of the $>2.5-\mathrm{MeV}$ electron rate and a rate due to $>6.5-\mathrm{MeV}$ electrons in this region. The rate ratio $\mathrm{BS} 4 /(>2.5 \mathrm{MeV} e)$ is thus roughly proportional to the flux of $>6.5-\mathrm{MeV}$ electrons. The broad minimum in the $>60-\mathrm{MeV}$ proton counting rate from $L=3.02$ to 3.14 marks the region swept-out by Mimas due to its orbital eccentricity, the Mimas macrosignature. Dashed lines in Figure $1 b$ mark the smaller electron absorption signature.
$\boldsymbol{R}_{\mathbf{S}}$. The size of this large-scale proton absorption signature is not related to the size of Mimas. Rather, it is due to Mimas's 0.02 orbital eccentricity. As a result, the radial diffusion of these protons must be negligible over the $\sim 11$ hours required for Mimas to move from its minimum to its maximum orbital radius. Thus, as recognized from the Pioneer 11 observations [Van Allen et al., 1980b; Simpson et al., 1980b; McDonald et al., 1980; Fillius and McIlwain, 1980], this minimum in the proton flux is a stable, stationary feature of the energetic proton flux in Saturn's inner magnetosphere. In contrast, the electron profiles show only smaller-scale fluctuations in intensity superimposed on an overall increase in flux intensity with decreasing distance to Saturn. No broad minimum is observed in the electron data of Figure 1 coincident with Mimas's orbit.

The difference in the large-scale 'macrosignature' of Mimas between electrons and protons is the result of the large difference between the drift periods of these two species relative to Mimas [Van Allen et al., 1980b]. Since the proton drift velocity is in the same direction as Saturn's rotational velocity, protons drift downstream in the corotational flow. Thus the drift period of protons relative to Mimas is always less than $\sim 20$ hours, Mimas's period relative to Saturn. The electron drift velocity is in the direction opposite to Saturn's rotational velocity, however. Electrons drift upstream, against corotation. Relative to Mimas these velocities are similar in magnitude. As a result, the drift velocity relative to Mimas is very small for electrons near $1 \mathrm{MeV}$. These electrons are rarely exposed to absorption by Mimas and thus show no significant largescale absorption signature. This electron resonance phenomenon and the differences in the frequency of encounters with Mimas between electrons and protons have been discussed in a number of papers [Thomsen and Van Allen, 1980; McDonald et al., 1980; Fillius and Mcllwain, 1980; Simpson et al., 1980b].

The large difference in the proton intensity between the inbound and outbound passes, a factor of 3 to 5 , resulted from the lower latitude of the outbound pass $\left(-6^{\circ}\right.$, as opposed to $+19^{\circ}$ inbound) and the fact that the proton flux pitch angle distribution is sharply peaked near $90^{\circ}$ [Schardt and McDonald, this issue]. Thus the proton flux is more intense nearer the equatorial plane. Beyond $L=3.2$ outbound, there is a small contribution from these protons in the $>5-\mathrm{MeV}$ and BS4 electron rates. Between $L=3.02$ and 3.14, however, these energetic protons did not contribute significantly to the electron rates in Figure 1.

From 0446 to 0448 of day 238, during the outbound pass (Figure $1 b$ ), a transient decrease was observed simultaneously in all of the electron rates. The depth of this electron signature and the detailed shape of the profile are energy dependent (Figure 2). The higher-energy rates show more evidence of a secondary minimum at 0446 . The separation between these two minima is $\Delta L \sim 0.02$, equivalent to an equatorial radial distance of $\sim 1200 \mathrm{~km}$. The full width at half of the minimum 


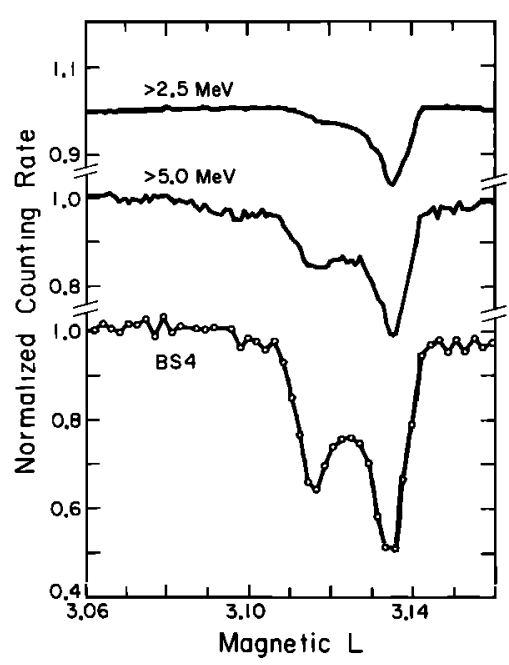

Fig. 2. A plot of the electron signatures from Figure $1 b$ on an expanded scale. These counting rates have been normalized to emphasize the differences between the signatures.

of the major decrease was $\sim 600 \mathrm{~km}$, somewhat greater than the effective geometrical sweeping diameter of Mimas $D_{M}=$ $2\left(r_{M}+2 r_{g}\right)=450 \mathrm{~km}$, where $r_{M}=195 \mathrm{~km}$ is the radius of Mimas [Smith et al., 1982], and $r_{g}=14 \mathrm{~km}$ is the gyroradius of $2.5 \mathrm{MeV}$ electrons at $L=3.13$. No comparable electron signature was observed during the inbound pass of Voyager 2 across this region (Figure 1a). The smaller fluctuations visible in the inbound $>5-\mathrm{MeV}$ and the BS4 rates may have resulted from the spacecraft roll maneuver taking place at this time which resulted in these detectors sampling different parts of the anisotropic electron pitch angle distribution in this region. No maneuvers were being executed during the outbound pass through the region in Figure 1.

The electron signatures of Figure $1 b$ are displayed in Figure 2 on an expanded and normalized scale. The profiles plotted in Figure 2 were calculated from the corresponding counting rates of Figure $1 b$ by dividing each rate by a function of the form $A \exp \left(-L / L_{0}\right)$. In each case, $A$ and $L_{0}$ were determined by a least squares best fit to each rate over the region $L=3.00$ to 3.16 but excluding the signature from $L=3.105$ to 3.145 .

Figure 2 illustrates the energy dependence of the shapes of these signatures. For the major decrease at $L=3.135$, the $>2.5-\mathrm{MeV}$ rate decreased by $17 \%$, while the $>5-\mathrm{MeV}$ rate decreased by $30 \%$. The $50 \%$ reduction in the BS4 rate coupled with the fact that the B2 rate decreased to $83 \%$ of its nominal value suggests that the $C 4$ rate $(>6.5 \mathrm{MeV})$ was reduced by $40 \%$ at $L=3.135$. Samples of the C4, C3, and C2 rates were obtained only once for each 16 samples of the $B 2$ rate. While the samples of these rates which were obtained are consistent with the other data, there are not enough of them to define the signatures in these rates by themselves. The secondary minimum at $L=3.115$ had a similar, but more dramatic, energy dependence. This feature appears as only a shoulder in the $>2.5-\mathrm{MeV}$ rate (3\% reduction), while in the $>5 .-\mathrm{MeV}$ rate there is a clear minimum (18\% reduction), and a comparison of the B2 and BS4 rates suggests that the C4 rate must have decreased by nearly $35 \%$ in this secondary minimum. Since the orientation of these detectors was fixed over the interval when the signature was observed, and since most of these detectors were sensitive to electrons over most of a full hemisphere, we cannot infer any pitch angle dependence of these signatures, and we prefer to attribute the differences between these signatures to an energy dependence.

The absence of any significant absorption signature in the inbound electron flux profiles led us to conclude in an earlier report [Vogt et al., 1982] that Mimas could not have been responsible for the outbound signature. In this report we now present a more complete analysis of these data, taking into account the remarkable electron spectrum characteristic of this region. We will again conclude that Mimas could not have produced the signature that we observed. In addition, our analysis permits us to place limits on the characteristics of any absorber which could have produced the signature as well as limits on the radial diffusion coefficient for electrons at the orbit of Mimas.

\section{Mimas Absorption Signature Calculation}

In the energy range to which the CRS instrument is sensitive, the primary effect of a satellite like Mimas orbiting within Saturn's magnetosphere will be to absorb the radiation incident on it. Due to the longitudinal motion (drift plus corotation with Saturn) of the radiation, any absorber will leave an absorption 'wake' on any $L$ shell it passes. The maximum possible longitudinal length of the wake for any particle energy or species is equal to the product of the energydependent drift rate of that particle type relative to the absorber and of the length of time that the absorber occupies the drift shell, assuming the absorber's orbit or the drift shell are eccentric. The fraction of particles absorbed within the wake is determined by the probability that a charged particle near the longitude of the absorber cannot 'leapfrog' or 'corkscrew' past the absorber via latitudinal bounce motion ( $R$. Rairden, unpublished manuscript, 1980). For electrons with the energies considered in this report this probability is negligible at Mimas. Thus Mimas absorbs virtually all of the electrons on magnetic field lines that it crosses. After the absorber passes an $L$ shell these wakes separate due to the energy dependence of the drift velocity. Thus to a detector with a broad energy response, the absorption signature will decay with time as older wakes are spread over a longer longitude range. In addition to this energy-dependent dispersion, the absorption signature will be dispersed by radial diffusion.

In an initial attempt to model the electron absorption signatures of Figure 1, we assume that they were produced by Mimas. The expected Mimas absorption signature profiles in the electron rates of Figure 1 are calculated from the local electron spectrum, and these profiles are compared to the observations. The effects of radial diffusion are neglected in this initial calculation.

Figure 3 illustrates the inbound trajectory of Voyager 2 in a coordinate system which corotates with Mimas. Due to its orbital eccentricity, Mimas oscillates at $0^{\circ}$ longitude between $L=3.02$ to 3.14 every 22.73 hours. In this coordinate system, electrons with energies $\geq 1-\mathrm{MeV}$ drift to the west (left to right) while lower-energy electrons and protons drift to the east (right to left). For any single electron energy, the absorption wake of Mimas is nearly a sinusoid in longitude, extending from Mimas with a 'wavelength' equal to $\omega_{D} \times 22.7$ hours where $\omega_{D}$ is the energy-dependent electron drift frequency relative to Mimas, as defined in the following section. Examples of such sinusoids are shown in Figure 3 for $2.9-\mathrm{MeV}$ electrons. An important characteristic of the Voyager 2 trajectory illustrated in Figure 3 is that the longitudes of the inbound and outbound legs, relative to Mimas, differ by only 


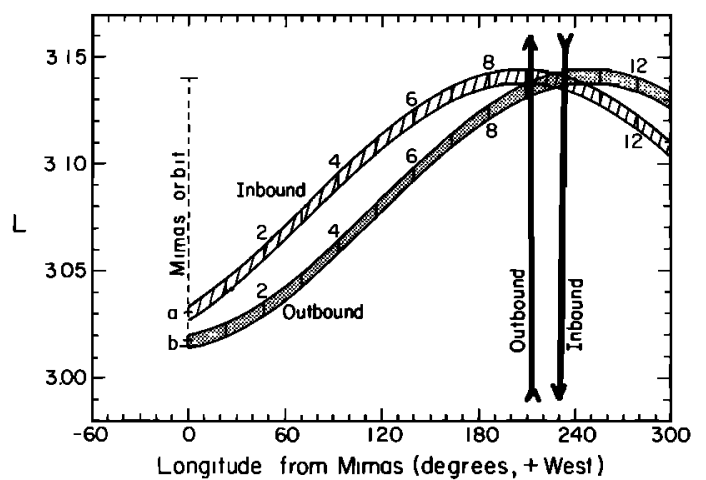

Fig. 3. The heavy solid lines illustrate the trajectory of Voyager 2 relative to Mimas in ( $L$, longitude) coordinates. The coordinate system rotates so that Mimas remains at $0^{\circ}$ longitude while oscillating between $L=3.02$ and 3.14. The sinusoidal bands extending to the right from the positions of Mimas at the times of the inbound (a) and outbound (b) passes illustrate where any absorption wake of Mimas in $2.9-\mathrm{MeV}$ electrons would be at the times that Voyager 2 crossed this region both inbound and outbound. (For 2.9-MeV electrons, Voyager 2 would have crossed the wake at the position of the observed electron signature.) Numbered tic marks along the wakes label the time in hours since that region passed Mimas. Similar wakes may be drawn for any other energy. The wavelength of the wake in this display would increase with increasing electron energy above the $1-\mathrm{MeV}$ resonant energy. Below the resonant energy the wakes extend to the left.

$\sim 10 \%\left(20^{\circ}\right.$ out of $\left.>200^{\circ}\right)$ when measured in the direction that energetic electrons drift. The inbound and outbound passages of Voyager 2 across this region were separated by 2 hours (see Figure 1).

The electron energy spectrum also must be known to determine the expected profile of Mimas's absorption signature. The remarkable electron spectrum in the vicinity of Mimas is illustrated in Figure 4, which shows the integral electron spectrum at $L=3.1$ as determined by the available Pioneer 11 and Voyager 2 measurements of the electron flux.

The hachured boxes in Figure 4 are flux measurements from the CRS instrument. They were calculated from counting rates of single detectors with different amounts of passive shielding and different electronic thresholds (Table 1). The size of the box at each energy reflects our estimate of the uncertainty of the electron energy threshold and of the effective geometrical factor. The measurement at $2.5 \mathrm{MeV}$ labeled with a small arrow may not have been fully corrected. This point was calculated from the counting rate of a 2-mm silicon detector with a $2.2-\mathrm{MeV}$ threshold. Due to the high threshold, the electron detection efficiency of this detector is small. The plotted value includes a correction for this efficiency (calculated to be a maximum of $10 \%$ ) using the method of Lupton and Stone [1972]. Since that method provides an upper limit to the detection efficiency, the corrected flux value is a lower limit. The fact that this value is inconsistent with the rest of the data suggests that the real efficiency may be significantly smaller.

The CRS counting rates in Mimas's orbit may confidently be attributed to electrons because, as illustrated in Figure 1, the proton flux is greatly reduced at $L=3.1$ by Mimas. Only the highest energy box at 9 . MeV could have a significant contribution from energetic protons. Thus it may be viewed as an upper limit to the electron flux. A detailed analysis of these counting rates [Schardt and McDonald, this issue] suggests that protons may be responsible for $70 \%$ of the observed counts in this $9-\mathrm{MeV}$ electron flux rate at $L=3.1$. Finally. since the counting rates $R$ on which the CRS flux measurements are based have different radial gradients $((1 / R)(\partial R / \partial L))$ in this region, gradients which are steeper with increasing energy (Table 1), and since the shape of the microsignature varies systematically with increasing electron energy (Figure 1), we believe that these counting rates truly measure different portions of the energy spectrum rather than, for example, pileup or bremstrahlung from a common, lower-energy flux source. The radial gradients of each rate across Mimas's orbit ( $L=3.02$ to 3.14 ) are nearly the same inbound and outbound (excluding the outbound signature), thus indicating that the instrument was measuring the same flux distribution along both legs of its trajectory in this region.

The other electron flux measurements displayed in Figure 4 were calculated from published electron counting rates and geometrical factors [Van Allen et al., 1980b; Fillius et al., 1980; Simpson et al., 1980b; Krimigis et al., 1982a] or from published differential fluxes [McDonald et al., 1980]. The point at $3.4 \mathrm{MeV}$ from Simpson et al. [1980b] is from their electron current detector (ECD). In view of the steepness of the electron spectrum and the response characteristics of the detector, the true equivalent energy threshold is likely less than 3.4 MeV (R. B. McKibben, private communication, 1982) so that this point would shift to the left in Figure 4 by perhaps as much as $1 \mathrm{MeV}$ (as indicated in Figure 4) if it were corrected for the shape of the spectrum. The $1 \mathrm{~g} \mathrm{~cm}^{-2}$ beryllium shield surrounding the ECD detector shields it from electrons with energies below $2 \mathrm{MeV}$.

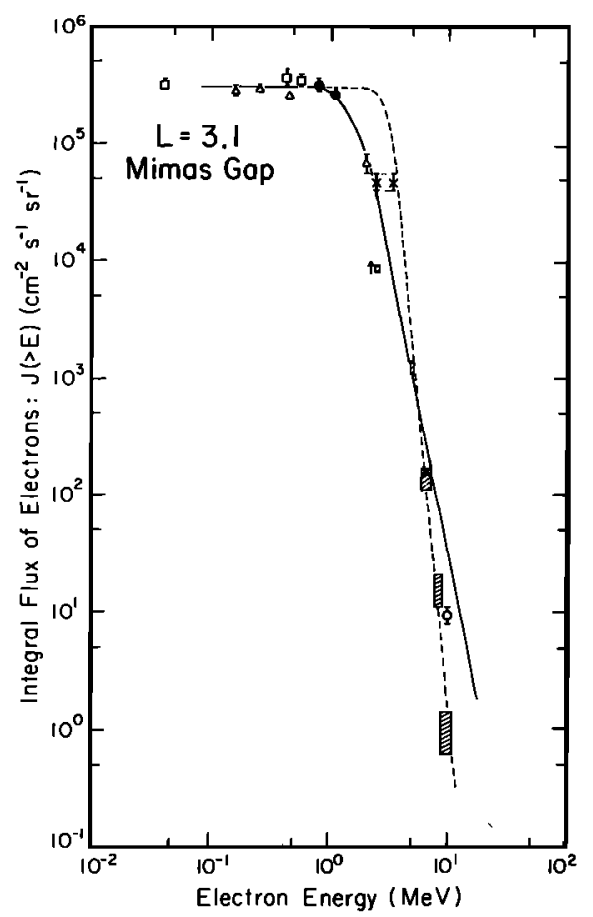

Fig. 4. The integral electron spectrum in the vicinity of Mimas. The hachured boxes are data calculated from the CRS instrument, and the open circle is from Krimigis et al. [1982a]. Both of these data sets were obtained from instruments aboard Voyager 2 in August 1981. Open squares are from Van Allen et al. [1980b], open triangles are from Fillius and McIlwain [1980], solid circles are from McDonald et al. [1980], and crosses are from Simpson et al. [1980b]. These are observations from the Pioneer 11 spacecraft taken in August 1979. Error bars assigned to points reflect the variability in the counting rates during both inbound and outbound passes. The dashed and solid lines indicate model spectra chosen as limiting cases of the true spectrum (see text). 
Qualitatively, the electron spectrum at $L=3.1$ is consistent with the model proposed by Van Allen et al. [1980b]. Since this integral spectrum is almost flat between $\sim 100 \mathrm{keV}$ and $\sim 1 \mathrm{MeV}$, and falls as $E^{-\gamma}$ with $\gamma=5$ to 10 above several $\mathrm{MeV}$, nearly all of the electrons above $\sim 100 \mathrm{keV}$ at $L=3.1$ have energies of 1 to $3 \mathrm{MeV}$. Van Allen et al. [1980b] have proposed that this form of the spectrum is produced by the 'band-pass filtering' action of Saturn's satellites on the inwardly diffusing electron population. The filtering action arises because electrons can have drift velocities that are comparable to a satellite's angular velocity. Near the resonant electron energy, i.e., where these two velocities are equal, the resonant electrons can diffuse across the satellite's orbit with a very small probability of being absorbed by the satellite. Thus the action of a satellite on an inwardly diffusing electron pop!llation is to pass preferentially electrons in a certain energy band. Through this mechanism, Enceladus passes electrons which arrive at Mimas with energies of $\sim 1.6 \mathrm{MeV}$ (assuming the conservation of the first two adiabatic invariants and equatorially mirroring electrons). While the data of Figure 4 are not precise enough to determine the characteristic energy to better than $1 \mathrm{MeV}$, they are consistent with $1.6 \mathrm{MeV}$, hence qualitatively consistent with the model. However, the bandpass filter model would not explain the increase in electron flux intensity at lower energies reported by Krimigis et al. [1982b].

Another conclusion of Van Allen et al., [1980b] concerning the electron spectrum at the orbit of Mimas, namely, that the spectrum is nearly monoenergetic with $\delta E \sim 0.1 \mathrm{MeV}$ about $1.6 \mathrm{MeV}$, is not supported by the data of Figure 4. Rather, the data of Figure 4 indicate that $20 \%$ or more of the electron flux above $0.1 \mathrm{MeV}$ is above $2 \mathrm{MeV}$. The $0.1-\mathrm{MeV}$ estimate of the width of the spectrum was not based on the 'band-pass filter' model but, rather, on the analysis of an electron absorption signature and the assumption that this signature was due to Mimas. If this Pioneer 11 signature was not due to Mimas [Simpson et al., 1980a, b], a conclusion that we support in a later section of this paper, then this constraint on the width of the electron energy spectrum is removed.

To use this electron spectrum in calculating the expected Mimas absorption signature, we have adopted the two models shown as the solid and dashed lines in Figure 4. The two models are adopted as probable limiting cases of the true spectrum to assess the effect on the calculation of changes in the spectrum. Both models are of the form

$$
J(>E)=\frac{J_{0}}{1+\left(E / E_{0}\right)^{y}}
$$

In case 1 (solid line), $\gamma=5$ and $E_{0}=1.6 \mathrm{MeV}$, while for case 2 (dashed line), $\gamma=10$ and $E_{0}=3.0 \mathrm{MeV}$. For both cases $J_{0}=$ $3 \times 10^{5}$ electrons $\mathrm{cm}^{-2} \mathrm{sr}^{-1} \mathrm{~s}^{-1}$, although this constant does not affect the calculation or the results in the rest of this paper.

\section{Expected Mimas Absorption Signature}

To calculate the expected absorption signature of Mimas from the spectra of Figure 4, the true orbit of Mimas (provided by the navigation team of the Jet Propulsion Laboratory) was converted to obtain the orbit in magnetic $L$ coordinates, $L_{M}(t)$. This function, together with the radius of Mimas, was used to construct the functions $t_{i}(L)$ and $t_{f}(L)$, which are the times when Mimas last entered $\left(t_{i}\right)$ and left $\left(t_{f}\right)$ the drift shell labeled by $L$. The expression for the angular drift velocity of

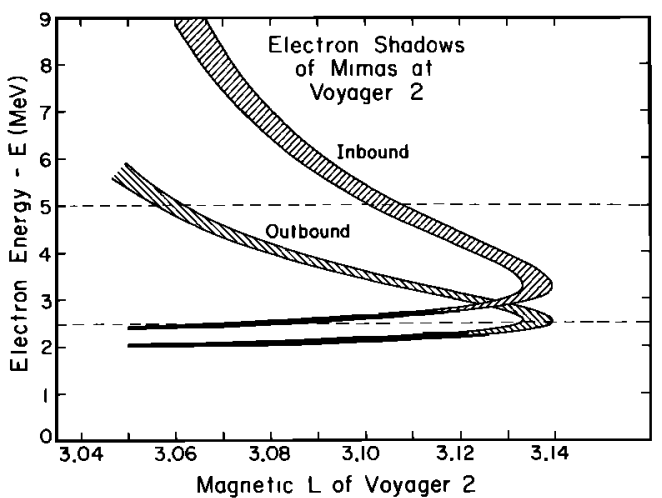

Fig. 5. The hachured regions labeled inbound and outbound indicate the range electron energies that would have been absorbed by Mimas during its most recent orbit as the Mimas absorption shadow crossed the position of Voyager 2. Horizontal dashed lines at 2.5 $\mathrm{MeV}$ and $5 \mathrm{MeV}$ indicate CRS counting rate thresholds. The higherenergy end of the hachured regions asymptotically approach the $L$ shell position of Mimas at the time Voyager 2 passed. The age of the signature increases with decreasing electron energy.

electrons of kinetic energy $E$ relative to Mimas which we used is based on the dipole approximation [see Thomsen and Van Allen, 1980, and references therein]:

$$
\omega_{D}=A L E \alpha(E)\left[F / G\left(\lambda_{m}\right)\right]-\Omega
$$

where $\alpha(E)=(E+2 m) /(E+m)$ is a relativistic factor which takes values between 1 and 1.2 for our data, $m$ is the electron rest mass, $F / G$ is a factor (between 0.9 and 1.0 for these data) which depends on a particle's mirror latitude, $\lambda_{m}$, and $\Omega=\omega_{M}$ $-\omega_{S}=8.70 \times 10^{-5} \mathrm{rad} \mathrm{s}^{-1}$ is the angular velocity of Mimas relative to Saturn's magnetic field, where $\omega_{M}=-7.68 \times 10^{-5}$ $\mathrm{rad} \mathrm{s}^{-1}$ is the inertial angular velocity of Mimas and $\omega_{s}=$ $-1.638 \times 10^{-4} \mathrm{rad} \mathrm{s}^{-1}$ is the magnetic (SLS) rotation rate of Saturn [Desch and Kaiser, 1981]. The constant $A=1.96 \times$ $10^{-5} \mathrm{rad} \mathrm{MeV}^{-1} \mathrm{~s}^{-1}$ differs slightly from that used by Thomsen and Van Allen [1980] due to revised values for the nominal equatorial radius of Saturn $(60,330 \mathrm{~km}$ rather than 60,000 $\mathrm{km})$ and for Saturn's dipole moment $\left(0.21 \mathrm{G} \boldsymbol{R}_{S}{ }^{3}\right.$ rather than $0.2 \mathrm{G} \boldsymbol{R}_{s}{ }^{3}$ ) [Ness et al., 1982]. For electrons with energies $E>1.1 \mathrm{MeV}$ the first term of (2) is the larger. Thus $\omega_{D}>0$, where, for the purposes of this paper, the positive angular direction is from east to west (clockwise as viewed from the north of Saturn). If the inbound or outbound legs of the Voyager 2 trajectory are expressed in Mimas-fixed coordinates, i.e., a coordinate system that corotates with Mimas, in the form $\varphi(t), L(t), \lambda(t)$, where $\varphi$ is the longitude angle from Mimas to Voyager and $\lambda$ is the latitude of the spacecraft, then at any time $t$ along the Voyager 2 trajectory in the region of $L$ swept by Mimas, electrons with energies $E$ such that $E_{i}<$ $E<E_{f}$ will have been absorbed, where

$$
\begin{gathered}
\omega_{D}\left(E_{i}\right)=\frac{\varphi(t)}{t-t_{i}[L(t)]} \\
\omega_{D}\left(E_{f}\right)=\frac{\varphi(t)}{t-t_{f}[L(t)]}
\end{gathered}
$$

The resulting shadow of Mimas in the electron energy spectrum along the Voyager 2 trajectory, both inbound and outbound, is plotted in Figure 5. The shaded regions of Figure 5 correspond to the regions $E_{i}<E<E_{f}$ that Mimas produced during the $\sim 20$ hours prior the passage of Voyager 2 . The normalized absorption signature for each rate (neglecting dif- 


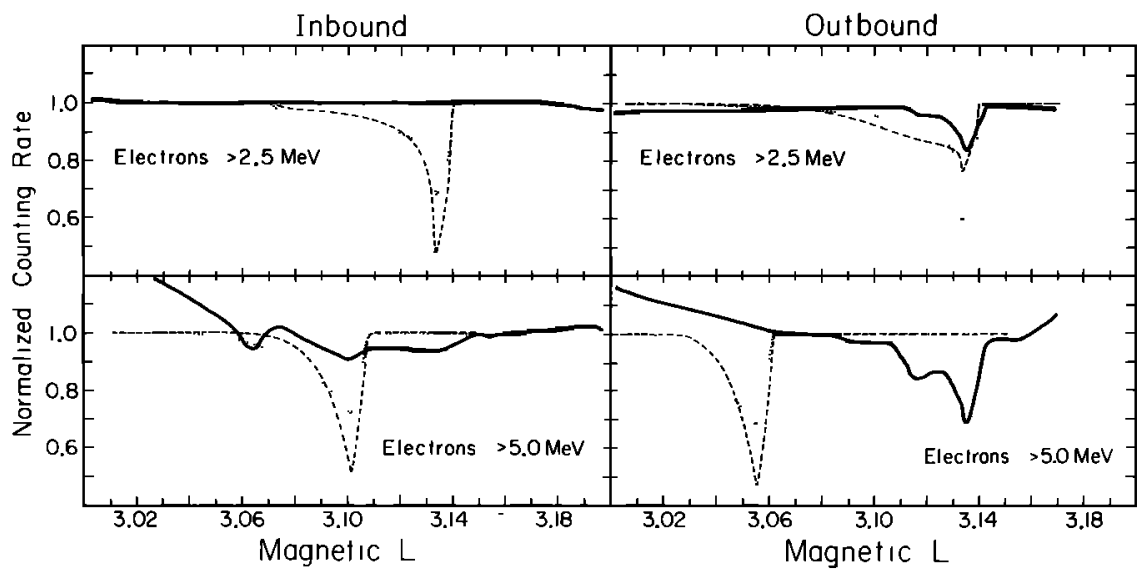

Fig. 6. A comparison between the electron microsignatures observed on Voyager 2 and the microsignatures that would be expected from Mimas, neglecting radial diffusion. The solid curve of each panel is the normalized rate of $>2.5-\mathrm{MeV}$ electrons (upper panels) and $>5.0-\mathrm{MeV}$ electrons (lower panels) both inbound (left panels) and outbound (right panels) across the orbit of Mimas. The dashed and dotted lines in each panel are the microabsorption signatures due to Mimas that would be expected neglecting the effects of radial diffusion. The dotted curve was calculated using the model spectrum displayed as the solid line in Figure 4, and the dashed curve corresponds to the dashed-line model spectrum.

fusion) is calculated by integrating the spectra of Figure 4 above the rate energy threshold, but excluding the region from $E_{i}$ to $E_{f}$.

If the effects of radial diffusion were negligible over the $\sim 10$-hour interval required for $\sim 2.5-\mathrm{MeV}$ electrons to drift from Mimas to the position of Voyager 2, the absorption signatures observed by the CRS instrument should have resembled the dashed or dotted curves drawn in Figure 6. The solid line drawn in each panel of Figure 6 is the measured $>2.5-\mathrm{MeV}$ or $>5-\mathrm{MeV}$ counting rate which has been normalized by dividing the observed rate by its least squares best fit exponential in $L$. The signatures from $L=3.105$ to 3.145 were excluded from these fits. The dashed lines are the signatures calculated using the model spectrum with $E_{0}=3.0 \mathrm{MeV}$ and $\alpha=10$ (the dashed-line spectrum of Figure 4), while the dotted lines are based on the $E_{0}=1.6 \mathrm{MeV}$ and $\alpha=5$ model spectrum (the solid line of Figure 4).

Even though the real electron spectrum may differ in detail from these model spectra, these calculations indicate that the qualitative characteristics of the absorption signatures are not sensitive to the exact form of the electron spectrum. However, there are quantitative differences between the calculated absorption signatures of Figure 4 which result from differences between these model spectra. An examination of the origin of these differences provides insight into the nature of such signatures. For the $>5-\mathrm{MeV}$ rate, the dashed-line signature is deeper and narrower because with this steeper spectrum more of the counts are from electrons just above the detector threshold. The calculated outbound signature is generally deeper than the inbound signature because the spacecraft passed closer to Mimas along the outbound pass and thus the signature was 'fresher'; it spanned a larger energy range. For the $>2.5-\mathrm{MeV}$ rate and the $E_{0}=3.0 \mathrm{MeV}$ spectrum, however, the calculated inbound signature is deeper. This is due to the combination of two effects, both of which are illustrated in Figure 5. First, for both the inbound and the outbound passes the maximum calculated absorption above $2.5 \mathrm{MeV}$ occurred at the maximum radial excursion of Mimas, the region where Mimas spends the most time at the same $L$, resulting in the longest (in longitude) or widest (in energy) absorption wake. Second, for the inbound pass this wake spanned 3.0 MeV, whereas for the outbound pass this wake extended to only $2.8 \mathrm{MeV}$. Thus the calculated inbound wake is deeper since for the $E_{0}=3.0 \mathrm{MeV}$ spectrum, most of the flux is concentrated near $3.0 \mathrm{MeV}$.

As Figure 6 illustrates, the outbound $>2.5-\mathrm{MeV}$ signature was observed at the location where a Mimas signature would have been expected. However, if that signature were due to Mimas, the $>5.0-\mathrm{MeV}$ Mimas signature should not have been coincident with the $>2.5-\mathrm{MeV}$ signature, rather the $>5.0-\mathrm{MeV}$ signature should have appeared at $L \approx 3.05$ due to the energy dispersion of the electron drift velocity. The signature in the $>5.0-\mathrm{MeV}$ rate should appear closer to Mimas's current position because higher-energy electrons drift more rapidly. Thus we conclude that it was entirely fortuitous that a $2.5-\mathrm{MeV}$ signature was observed where Mimas's signature was expected. Second, and perhaps more convincingly, if the effect of radial diffusion were small enough to be neglected (i.e., if the diffusion coefficient $D$ were smaller than $10^{-10} R_{S}{ }^{2}$ $\mathrm{s}^{-1}$ ), then absorption signatures due to Mimas should have been observed on both the inbound and outbound passes. Thus we conclude not only that Mimas could not have produced the observed signature, but also that no absorption signature due to Mimas was observable. Since Mimas cannot have produced the observed signature, we are motivated to search for another cause for it. Since no absorption signature due to Mimas was observable, we can calculate a lower limit for the radial diffusion coefficient of $\mathrm{MeV}$ electrons at $L \sim 3.1$.

\section{Limits on the Radial Diffusion Coefficient}

To estimate the importance of the effect of radial diffusion on a satellite absorption signature, it is convenient to use a normalized diffusion time $\tau$ defined as

$$
\tau=4 D t / b^{2}
$$

where $D$ is the diffusion coefficient, $t$ is the age of the signature, and $b$ is the radial size of the original signature. This normalized time is convenient because the maximum fractional depth $Z$ of an absorption signature in a one- 
dimensional diffusion model like the model used by Van Allen et al. [1980b] is given by

$$
Z=\operatorname{erf}\left(\tau^{-1 / 2}\right)
$$

where erf $(\quad)$ is the error function. For the case of electron absorption by Mimas, $t$ is the drift time from Mimas to the spacecraft and $b$ is the effective radius of Mimas for absorbing electrons (see section 2).

In their analysis of electron data in the vicinity of Mimas, Van Allen et al. [1980b] estimated the radial diffusion coefficient $D$ at $L=3.1$ to be in the range $8 \times 10^{-12}$ to $4 \times 10^{-11}$ $R_{S}{ }^{2} \mathrm{~s}^{-1}$. If this value for $D$ were correct, then for the $>2.5-\mathrm{MeV}$ electrons observed at Voyager $2, \tau$ would have had a value less than 1 both inbound and outbound. If $\tau<1$, then $Z>0.8$, thus the signatures should have been at least $\sim 80 \%$ as deep as the calculated signatures of Figure 6 . Since any Mimas absorption signature along the Voyager 2 inbound pass must have been very small, the real diffusion coefficient must be much larger.

The lower bound on the radial diffusion coefficient for $\mathrm{MeV}$ electrons at $L=3.1$ that we obtain from these Voyager 2 data is similarly based on the one-dimensional diffusion model. The fluctuations in the normalized inbound $>2.5-\mathrm{MeV}$ electron rate are $<5 \%$ Since in the absence of radial diffusion a $30-50 \%$ deep signature would have been expected, we estimate a limit on $Z$ of $Z \leq 0.1$, thus $\tau \geq 100$. Using $b=220 \mathrm{~km}$ for the effective radius of Mimas and $t=7.5$ hours as the drift time from Mimas to Voyager, the estimate for a lower limit on the radial diffusion coefficient which results is $D>10^{-8} R_{S}{ }^{2} \mathrm{~s}^{-1}$. This estimate is consistent with the value obtained by Simpson et al. [1980b] for $\sim 10$ MeV-electrons of $4 \times 10^{-8} R_{s}^{2} s^{-1}$, which was inferred from the depth of the large-scale electron macrosignature that results from Mimas's orbital eccentricity.

\section{Possible Causes for the Observed Signature}

Since Mimas cannot have produced the small-scale electron absorption signature observed by Voyager 2, we are led to search for other possible causes. Several candidates are examined in this section. Compression of the magnetic field could produce a dip in the observed electron flux through the combined effects of the steep electron energy spectrum and a radial gradient in the flux intensity. This mechanism may be reversible in the sense that few electrons are lost from the region. When the fluctuation passes, the region returns to its original state. Alternatively, an absorption signature could be produced by intense, localized (in $L$ and perhaps longitude) wave-particle interactions which dump the electrons into Saturn's atmosphere. The final possibility that we consider is absorption by additional material sharing Mimas's orbit in or near Saturn's equatorial plane.

\subsection{Magnetic Fluctuations and Turbulence}

Barfield et al. [1971], in a study of compressional micropulsations, provide a formula for the expected change in flux intensity, $J$, for equatorially mirroring particles given a fluctuation in the local magnetic field magnitude, $B_{f}$, the particle energy spectrum, and the radial particle gradient:

$$
\begin{aligned}
\left.\frac{\partial \ln J}{\partial \ln B_{f}}\right|_{E}=\left\{1-\left(\frac{\gamma+1}{2 \gamma}\right)\right. & \left.\left.\frac{\partial \ln J}{\partial \ln E}\right|_{B}\right\} \frac{d \ln B_{c}}{d \ln B_{f}} \\
& +\left.\frac{\partial \ln J}{\partial \ln B_{c}}\right|_{E}\left(1-\frac{d \ln B_{c}}{d \ln B_{f}}\right)
\end{aligned}
$$

In this formula, $\boldsymbol{B}_{\boldsymbol{f}}$ is the magnetic field intensity measured by a fixed observer, while $B_{c}$ is the field intensity experienced by a particle's guiding center, which moves with the fluctuation; $\gamma$ is the relativistic Lorentz factor. While this formula is only strictly applicable to equatorially mirroring electrons, it should provide an upper limit estimate for other pitch angles as long as the pitch angle distribution is isotropic or peaked at $90^{\circ}$ pitch angle. If we adopt the symmetric compression model of Roederer [1970], then $\partial \ln B_{c} / \partial \ln B_{f}=5 / 2$. The spectral index, $\partial \ln J / \partial \ln E$ is taken to be $S$ to 12 (Figure 4) and the factor $\partial \ln j / \partial \ln B_{c}=-L g_{r} / 3$, where $g_{r}=\partial \ln J / \partial L$ is the radial gradient which was observed to be in the range $g_{r}=$ -1.0 for the $>2.5-\mathrm{MeV}$ electron rate and $g_{r}=-4.7$ for the $>$ 5.0-MeV rate (Table 1). Thus we obtain

$$
\Delta J / J=\{2-20\} \Delta B / B
$$

In the region of interest, 48-s averages of the observed magnetic field (N. F. Ness, personal communication, 1982) show no fluctuations larger than $\Delta B / B=0.001,(\Delta B<1 \gamma)$, and an inspection of finer time resolution data confirms this conclusion (J. E. P. Connerney, personal communication, 1982). Thus on the basis of this analysis, no adiabatic magnetic fluctuation was observed which was large enough to produce the observed $(>15 \%)$ decrease in electron flux intensity.

A localized region of intense wave-particle interactions could also produce an absorption signature similar to that observed by scattering trapped electrons into Saturn's atmosphere. At $L=3.1$ the atmospheric loss cone extends to $\sim 8^{\circ}$ equatorial pitch angle and thus occupies $1 \%$ of the total solid angle. Thus at most, $1 \%$ of the particles in a flux tube can be scattered into the atmosphere in the time required for a particle to travel from the equator to its mirror latitude, a time interval equal to one quarter of the particle's bounce period, or $0.6 \mathrm{~s}$ for relativistic particles at $L=3.1$. In this strong pitch angle diffusion limit, therefore, it would take a 10-s burst of intense wave-particle activity to remove $15 \%$ of the electrons in a flux tube.

A problem with this hypothesis is that of confining the interaction to a region $\delta L \sim 0.02$ to produce a localized signature. Presumably, such confinement could be produced by spatial variations in the plasma density to create a duct in which the interactions occur. While whistler mode turbulence which could precipitate energetic electrons was observed [Scarf et al., 1982], these waves were observed throughout large regions of Saturn's inner magnetosphere. This electron signature, however, was a unique event. Data from the Voyager 2 plasma wave experiment were examined for any signals that could have caused a short-lived dropout in $\mathrm{MeV}$ electrons. None were observed through a large region surrounding the absorption signature (W. S. Kurth, personal communication, 1982).

\subsection{Electron Absorption by Material in Mimas's Orbit}

The final alternative that we consider for producing the signature is absorption by material in orbit with Mimas. There are several characteristics of the observations which make this an attractive possibility. First, this was the only such signature observed by the CRS instruments inside of the orbit of Enceladus, and it occurred in the orbital range of Mimas. Stable orbits exists for smaller particles near the orbits of larger satellites [Dermott et al., 1980]. Second, the width of the observed signature, $\Delta L \sim 0.02=1200 \mathrm{~km}$ is less than the maximum width of a 'ring' of particles that Mimas 
can sustain. According to the model of Dermott et al., this width is

$$
W=4 a(\alpha / 3)^{1 / 2}(m / M)^{1 / 3} \approx 1800 \mathrm{~km}
$$

where $\alpha \leq 1.2$ is a parameter, $m / M=6.7 \times 10^{-8}$ is the ratio of the mass of Mimas to the mass of Saturn, and $a=1.86$ $\times 10^{5} \mathrm{~km}$ is the semimajor axis of Mimas's orbit. Companion material is not uncommon at Saturn. Both Dione and Tethys have small co-orbital satellites [Smith et al., 1982]. Thus it is possible that material may also exist in orbit with Mimas.

Characteristics of the absorption signature suggest that the absorber must have been relatively close to the longitude of the spacecraft when the signature was observed. Any material in a Keplerian orbit of $3.1 R_{S}$ would have only been $20^{\circ}$ farther west of the spacecraft during the inbound pass than it was during the outbound pass (Figure 3). Since no significant absorption signature was observed inbound, this $20^{\circ}$ difference between the approach distances inbound and outbound measures the longitudinal extent of a signature. Thus the lifetime against diffusion must be of the order of the time required for electrons to drift through $20^{\circ}$, i.e., less than 2 hours, and the spacecraft must have passed within $20^{\circ}$ longitude of the absorber's position along the outbound pass. The lower bound on the diffusion coefficient that results from these considerations is estimated below. The lack of dispersion between the $>2.5-\mathrm{MeV}$ and $>5.0-\mathrm{MeV}$ signatures requires a similarly close approach distance outbound, assuming the orbit of the absorbing material shares Mimas's eccentricity.

Under the hypothesis that the $>17 \%$ deep electron microsignature observed by Voyager 2 outbound is due to absorption by orbiting material, since the absorption signature was not observed along the inbound pass ( $<5 \%$ absorption), another lower bound on the diffusion coefficient for $\mathrm{MeV}$ electrons at $L=3.1$ may be calculated which is independent of the estimate obtained earlier in section 5 . From the ratios of the depths of the inbound and outbound signatures, $Z=$ erf $\left(\tau^{-1 / 2}\right)<0.3$, which requires $\tau=4 D t / b^{2}>10$. The value for $t$ is the time required for $2.5-\mathrm{MeV}$ electrons to drift $20^{\circ}$ from an orbiting absorber at $3.1 R_{S}, 4 \times 10^{3} \mathrm{~s}$. The value for $b$ is the half width of the signature at half maximum (section 2, Figure 2), $300 \mathrm{~km}$ or $0.005 \boldsymbol{R}_{\mathbf{S}}$. Combining these quantities, we obtain another lower limit on the diffusion coefficient of $D>1.5$ $\times 10^{-8} R_{s}^{2} s^{-1}$. It is fortuitous that this value is so close to the other lower bound, $D>10^{-8} R_{s}{ }^{2} \mathrm{~s}^{-1}$ which was calculated in section 5 . However, the similarity between these results, which are based on entirely different considerations and thus are independent, gives added confidence in both the estimate of the diffusion coefficient and in the suggestion that the outbound Voyager 2 signature was due to absorption by material. If, for example, the real diffusion coefficient were as small as $10^{-10} R_{\mathrm{S}}{ }^{2} \mathrm{~s}^{-1}$, a significant inbound signature should have been observed.

The depth and width of an absorption signature are related to the size of the absorber. The absorber cannot be significantly larger than the width of the signature. Conversely, if the signature is wider than the absorber, the depth of the signature must be correspondingly smaller. For example, if the absorber were smaller than a few tens of kilometers, then by the time the signature had spread to $1000 \mathrm{~km}$ across (via radial diffusion) the maximum depth of the signature could not exceed a few percent. Thus to have a $10 \%$ or more absorption signature extending over $1000 \mathrm{~km}$ would require an object with a diameter of $100 \mathrm{~km}$ or more.

Following our initial report of these observations, Voyager
2 imaging frames of the region where we predicted the absorber to be located were examined. No objects were observed in these frames, and an upper limit of $10 \mathrm{~km}$ was obtained for the maximum size of any single object in this region with an albedo close to unity (S. P. Synnott, private communication, 1982). In order to reconcile this result with the hypothesis that the signature was due to absorbing material, either the absorber must be very dark, or else the absorber must consist of a cloud of small particles which may be brighter. The former possibility is very unlikely because it would require a $100 \mathrm{~km}$ object to have an albedo $<0.01$, making it much darker even than Phoebe or the dark side of Iapetus, which have albedos of $\sim 0.05$ in contrast to the 0.5 albedo of Mimas [Smith et al., 1982].

A cloud or swarm of small particles could fill an area (projected onto the equatorial plane) of a few $\times 10^{4} \mathrm{~km}^{2}$ and yet have a total area not larger than the area of a single $10-\mathrm{km}$ object. In order to absorb electrons with energies of a few $\mathrm{MeV}$ the sizes of the particles in the swarm must be at least a few centimeters. Since at least $30 \%$ of the $>5.0-\mathrm{MeV}$ flux was absorbed, in terms of this model

$$
a \rho n / \cos \alpha>-\ln (0.7)=0.36
$$

where $a$ is the area of an individual particle in the swarm, $\rho$ is the packing density of these particles projected onto the equatorial plane, $n$ is the number of times an electron penetrates the cloud, and $\alpha$ is the electron's equatorial pitch angle $\left(\alpha<75^{\circ}, \cos \alpha>0.26\right.$, for electrons which mirror at or above the latitude of Voyager 2). Due to the combined drift and bounce motion, $2.5-\mathrm{MeV}$ electrons would cross this cloud at the equatorial plane every $\sim 10 \mathrm{~km}$, while $5-\mathrm{MeV}$ electrons would cross every $\sim 40 \mathrm{~km}$. Thus for a cloud extending a few hundred kilometers in longitude, $n \approx 10$. Combining these estimates, the observations would require $a \rho \geq 0.01$, i.e., more than $1 \%$ of the total area of the cloud must be occulted by material.

In terms of this absorption model, the spatial structure in the electron signatures (Figure 2) must reflect a spatial variation in the density of the cloud. The energy dependence of the electron signatures, however, is more difficult to understand. Since the path length of a particle in one pass through a slab region (thinnest normal to the equator) that is large compared to the particle's gyroradius depends only on that particle's pitch angle not on its gyroradius and since higher-energy electrons, due to their larger drift velocities, would traverse such a slab fewer times, more lower-energy electrons (down to $\sim 1 \mathrm{MeV}$ ) should be absorbed. The measured signatures are deeper at higher energies, however. This contradiction may be reconcilable if the higher-energy electrons had flatter pitch angle distributions and thus were better confined to the equator where the absorber is presumed to be.

\section{Discussion and Summary}

The electron microsignature observed by the CRS instrument near Mimas may be explainable either as the result of an intense, localized burst of waves which scatter electrons out of the region or as the result of absorption by some additional material in Mimas's orbit. Both hypotheses require additional ad hoc assumptions to produce the absorption: a wave duct for the former, and a cloud of finer material for the latter; and neither set of assumptions has been directly verified by other observations. However, other observations of another microsignature, which is interpreted as due to low-energy ions [Carbary et al., this issue], were obtained simultaneously with the 
CRS electron signature. If the interpretation of these other data is correct, the ion observations discriminate against the wave-particle interaction hypothesis in favor of the absorption hypothesis.

Carbary et al. [this issue] report observing a microsignature similar to and simultaneously with the CRS signature but in ions with energies of $28-100 \mathrm{keV}$. In addition, they have measurements that suggest that the absorption is greatest for local pitch angles near $90^{\circ}$. These observations discriminate against the wave-particle mechanism for three reasons. First, since the gyrofrequencies of these ions and $\mathrm{MeV}$ electrons differ by a factor of 100 , the wave turbulence would need to be extremely broadband to interact resonantly with both of these particle species. Second, since the bounce period for these jons is 100 times longer than the relativistic electron bounce period, the required interaction time would rise from $6 \mathrm{~s}$ (estimated for the electrons) to many minutes. Finally, waves would be expected to make the particle distribution isotropic with particles lost at small pitch angles, in the loss cone, not at $90^{\circ}$ pitch angle. Alternatively, the pitch angle dependence of these ion observations supports the hypothesis of absorption by material. As reflected by the $\cos \alpha$ factor in (7), particles with larger pitch angles spend relatively more time in the absorption region and thus would be expected to be more heavily absorbed.

The simultaneous observation of signatures in both $\mathrm{MeV}$ electrons and $\mathrm{keV}$ ions also places stringent constraints on the location where the absorption must have occurred. Since these two populations of particles drift in opposite directions with a relative velocity of $60 \mathrm{~km} \mathrm{~s}^{-1}$ (for 5-MeV electrons and 100 $\mathrm{keV}$ ions), the observation of an absorption signature simultaneously in both ions and electrons means that the spacecraft must have crossed the $L$ shell of the interaction region at the longitude where the interaction was occurring and that the absorption was taking place as the spacecraft passed. The probability of such a close encounter is $\approx 10^{-4}$, assuming a single cloud $100 \mathrm{~km}$ long. Thus either the observation was extremely lucky or there may be many such clouds of particles in orbit with Mimas.

A similar set of circumstances surrounds the Pioneer 11 microsignature observations. Simpson et al. [1980b] reported simultaneous proton and electron signatures. Van Allen et al. [1980b] have disputed this report, suggesting that the proton signature was spurious, i.e., that it was produced by electrons. The probability that an absorption signature would have been observed along the inbound pass of Pioneer 11 may be expected to be higher than $10^{-4}$, the probability of crossing a $100-\mathrm{km}$ object or cloud randomly distributed around $3.1 R_{S}$, because Pioneer 11 passed near the Lagrangian point $\sim 60^{\circ}$ behind Mimas [Simpson et al., 1980b]. Small objects may be expected to reside in stable orbits in this region [Dermott et al., 1980]. The Voyager 2 absorption signature was not observed near a stable Lagrangian point, rather, it was observed $212^{\circ}$ behind (i.e., west of) Mimas. Thus if both the Voyager 2 and the Pioneer 11 signatures were produced as absorption signatures due to additional material in orbit with Mimas, it is improbable that the two signatures were produced by the same object or clump of material.

From the perspective of the Pioneer 11 and Voyager 2 charged particle observations, as currently interpreted, since microsignatures were observed in two of the four passes across the orbit of Mimas, and since the probability of an encounter with a single absorber is small, there must be a significant abundance of material surrounding Mimas. Clearly, the best way to support or refute this conclusion is through a detailed analysis of all available Voyager images of Mimas's orbit. Such analysis should either find some of the suspected objects or place upper limits on the sizes of any such absorbers that can be compared to the results of the particle absorption studies.

The other major results of the analysis presented in this report are independent of the question of what produced these signatures. The electron spectrum near Mimas's orbit as displayed in Figure 3 illustrates that most of the electron flux above $\sim 100 \mathrm{keV}$ is concentrated in the energy range 1-3 $\mathrm{MeV}$. Van Allen et al. [1980b] had predicted that the flux should be concentrated near $1.6 \mathrm{MeV}$ by the "band-pass filtering' action of Enceladus. The data of Figure 3 support this model. However, the observed spectrum is clearly broader than $\sim 0.1 \mathrm{MeV}$, the maximum width deduced by Van Allen et al. [1980b], who assumed that the Pioneer 11 signature was produced by Mimas. In their 'band-pass filter' model, Van Allen et al. further suggested that such a narrow spectrum would require a small diffusion coeflicient. They estimated a value of $D \approx 10^{-10} R_{s^{2}} s^{-1}$ at the orbit of Enceladus, which they extrapolated to $D \approx 8 \times 10^{-12}$ to $4 \times 10^{-11} R_{S}{ }^{2} s^{-1}$ at the orbit of Mimas.

However, the lower limit derived in this paper for the radial diffusion coefficient for electrons at Mimas's orbit is $D \geq 10^{-8}$ $R_{s}^{2} s^{-1}$. This value is based on the absence of any significant absorption signature in the inbound Voyager 2 data and on the lack of energy dispersion in the position of the outbound signature. Both of these features suggest that Mimas could not have produced the signature observed on Voyager 2. A similar limit on $D$ is required under the hypothesis that the signature is an absorption signature, in order to confine the signature to within $\sim 20^{\circ}$ of the absorber.

If this value for the diffusion coefficient is applied to the Pioneer 11 observations, it appears unlikely that the Pioneer 11 signature could be attributed to Mimas. Pioneer 11 passed $\sim 60^{\circ}$ from Mimas during its inbound pass [Simpson et al., $1980 b$; Van Allen et al., 1980b]. The age of the signature, i.e., the time since Mimas last passed the $L$ shell where the signature was observed, was 6.44 hours [Van Allen et al., 1980b]. Thus with our lower limit estimate for the diffusion coefficient, the normalized diffusion time (section 5) is $\tau \geq 70$, and the maximum depth of an absorption signature at any energy could be no more than $13 \%$. The dispersive effects of the energy dependence of the drift velocity would act to reduce the depth of a signature observed with a detector with a broad energy response even further. Since Van Allen et al. [1980b] observed absorption signatures with depths of $30-40 \%$, these signatures could not have been produced by Mimas if $D \geq$ $10^{-8} R_{s}^{2} s^{-1}$.

The lower limit for the electron diffusion coeflicient that we have obtained is significantly larger than the values inferred from studies of the high-energy proton population. Cooper [1983] has determined that $D \approx 10^{-15} L^{9} R_{s}{ }^{2} \mathrm{~s}^{-1}$ with an estimated uncertainty of a factor of 2 for $>30-\mathrm{MeV}$ protons. Thus at $L=3.1, D \approx 2.6 \times 10^{-11} R_{s}^{2} \mathrm{~s}^{-1}$. Van Allen [1983] has obtained $D \approx 2.8 \times 10^{-11} R_{s^{2}} \mathrm{~s}^{-1}$ for $>80-\mathrm{MeV}$ protons at $L=2.67$ with a lower limit of roughly half this value and an upper limit $\sim 25$ times larger. These results are all less than $1 \%$ of the lower limit inferred in this paper for $\mathrm{MeV}$ electrons. This difference may ultimately be reconcilable if the diffusion coefficient is inversely proportional to the rigidity of a particle or inversely proportional to the square of a particle's magnetic moment. It is important to note, however, that while the 
proton diffusion coefficient may be sensitive to phenomena that are effective over time scales of up to years (the inferred lifetime of high-energy protons in this region), the phenomena responsible for the electron diffusion analyzed in this paper operate on time scales of a few hours or less.

In conclusion, we emphasize two important aspects of this analysis. First, the limit inferred for the electron diffusion coefficient $D$ is independent of the question of what produced the observed signature. This limit is one of the principal results of this paper. Second, the $1 \%$ or more opacity inferred for the cloud on the basis of the absorption hypothesis is large, and a significant number of such clouds is suggested by combining the Voyager and Pioneer observations. While a more satisfactory explanation for these microsignature observations has not yet been suggested, these conclusions need to be either confirmed or refuted by analysis of imaging observations. Until that happens, the Mimas ghost will remain an enigma.

Acknowledgments. We greatly appreciate the efforts of R. E. Vogt and other members of the team from Caltech, Goddard Space Flight Center, the University of Arizona, and the University of New Hampshire who were responsible for the success of the CRS investigation. Useful discussions with A. W. Schardt and M. Schulz are gratefully acknowledged. We thank the Voyager Navigation Team of the Jet Propulsion Laboratory for providing accurate positions of the satellites of Saturn. This work was supported by NASA under NAS7-918 and NGR 05-002-160. DLC acknowledges additional support by the Aerospace Corporation's sponsored research program.

The editor thanks K. R. Pyle and S. Krimigis for their assistance in evaluating this paper.

\section{REFERENCES}

Bagenal, F, and J. D. Sullivan, Direct plasma measurements in the Io torus and inner magnetosphere of Jupiter, J. Geophys. Res., 86, $8447,1981$.

Barfield, J. N., L. J. Lanzerotti, C. G. Maclennan, G. A. Paulikas, and M. Schulz, Quiettime observation of a coherent compressional Pc 4 micropulsation at synchronous altitude, J. Geophys. Res., 76, 5252, 1971.

Broadfoot, A. L., M. J. S. Belton, P. Z. Takacs, B. R. Sandel, D. E. Shemansky, J. B. Holberg, J. M. Ajello, S. K. Atreya, T. M. Donahue, H. W. Moos, J. L. Bertaux, J. E. Blamont, D. F. Strobel, J. C. McConnell, A. Dalgarno, R. Goody, and M. B. McElroy, Extreme ultraviolet observations from Voyager 1 encounter with Jupiter, Science, 204, 979, 1979.

Carbary, J. F., S. M. Krimigis, and W.-H. Ip, Energetic particle microsignatures of Saturn's satellites, J. Geophys. Res., this issue.

Chenette, D. L., and L. Davis, Jr., An analysis of the structure of Saturn's magnetic field using charged particle absorption signatures, J. Geophys. Res., 87, 5267, 1982.

Connerney, J. E. P., N. F. Ness, and M. H. Acuña, Zonal harmonic model of Saturn's magnetic field from Voyager 1 and 2 observations, Nature, 298, 44, 1982.

Cooper, J. F., Nuclear cascades in Saturn's rings: Cosmic ray albedo neutron decay and the origins of trapped protons in the inner magnetosphere, J. Geophys. Res., 88, 3945, 1983.

Dermott, S. F., C. D. Murray, and A. T. Sinclair, The narrow rings of Jupiter, Saturn, and Uranus, Nature, 284, 309, 1980.

Desch, M. D., and M. L. Kaiser, Voyager measurements of the rotation period of Saturn's magnetic field, Geophys. Res. Lett., 8, 253, 1981.

Fillius, W., and C. McIlwain, Very energetic protons in Saturn's radiation belt, J. Geophys. Res., 85, 5803, 1980.

Fillius, W. W.-H. Ip, and C. E. Mcllwain, Trapped radiation belts of Saturn: First look, Science, 207, 425, 1980.

Krimigis, S. M., T. P. Armstrong, W. I. Axford, C. O. Bostrom, G. Gloeckler, E. P. Keath, L. J. Lanzerotti, J. F. Carbary, D. C. Hamil- ton, and E. C. Roelof, Low-energy hot plasma and particles in Saturn's magnetosphere, Science, 215, 571, 1982a.

Krimigis, S. M., J. F. Carbary, E. P. Keath, and T. P. Armstrong, Energetic electron spectra in Saturn's magnetosphere (abstract), Eos Trans. $A G U, 63,1068,1982 b$.

Lupton, J. E., and E. C. Stone, Measurements of electron detection efficiencies in solid state detectors, Nucl. Instrum. Methods, 98, 189, 1972.

McDonald, F. B., A. W. Schardt, and J. H. Trainor, If you've seen one magnetosphere, you haven't seen them all: Energetic particle observations in the Saturn magnetosphere, J. Geophys. Res., 85, 5813, 1980.

Mead, G. D., and W. N. Hess, Jupiter's radiation belts and the sweeping effects of its satellites, J. Geophys. Res., 78, 2793, 1973.

Ness, N. F., M. H. Acuña, K. W. Behannon, L. F. Burlaga, J. E. P. Connerney, R. P. Lepping, and F. M. Neubauer, Magnetic field studies by Voyager 2: Preliminary results at Saturn, Science, 215, $558,1982$.

Roederer, J. G., Dynamics of Geomagnetically Trapped Radiation, pp. 80-81, Springer-Verlag, New York, 1970.

Scarf, F. L., D. A. Gurnett, W. S. Kurth, and R. L. Poynter, Voyager 2 plasma wave observations at Saturn, Science, 215, 587, 1982.

Schardt, A. W., and F. B. McDonald, The flux and source of energetic protons in Saturn's inner magnetosphere, $J$. Geophys. Res., this issue.

Simpson, J. A., T. S. Bastian, D. L. Chenette, G. A. Lentz, R. B. McKibben, K. R. Pyle, and A. J. Tuzzolino, Saturnian trapped radiation and its absorption by satellites and rings: The first results from Pioneer 11, Science, 207, 411, 1980a.

Simpson, J. A., T. S. Bastian, D. L. Chenette, R. B. McKibben, and K. R. Pyle, The trapped radiations of Saturn and their absorption by satellites and rings, J. Geophys. Res., 85, 5731, $1980 b$.

Smith, B. A., L. Soderblom, R. Batson, P. Bridges, J. Inge, H. Masursky, E. Shoemaker, R. Beebe, J. Boyce, G. Briggs, A. Bunker, S A. Collins, C. J. Hansen, T. V. Johnson, J. L. Mitchell, R. J. Terrile, A. F. Cook II, J. Cuzzi, J. B. Pollack, G. E. Danielson, A. P. Ingersoll, M. E. Davies, G. E. Hunt, D. Morrison, T. Owen, C. Sagan, J. Veverka, R. Strom, and V. E. Soumi, A new look at the Saturn system: The Voyager 2 images, Science, 215, 504, 1982.

Smith, E. J., L. Davis, Jr., D. E. Jones, P. J. Coleman, Jr., D. S. Colburn, P. Dyal, and C. P. Sonett, Saturn's magnetosphere and its interaction with the solar wind, J. Geophys. Res., 85, 5655, 1980.

Stone, E. C., R. E. Vogt, F. B. McDonald, B. J. Teegarden, J. H. Trainor, J. R. Jokipii, and W. R. Webber, Cosmic ray investigation for the Voyager missions: Energetic particle studies in the outer heliosphere-And beyond, Space Sci. Rev., 21, 355, 1977.

Thomsen, M. F., Jovian magnetosphere-satellite interactions: Aspects of energetic charged particle loss, Rev. Geophys. Space Phys., 17, $369,1979$.

Thomsen, M. F., and J. A. Van Allen, Motions of trapped electrons and protons in Saturn's inner magnetosphere, J. Geophys. Res., 85 , $5831,1980$.

Trainor, J. H., F. B. McDonald, and A. W. Schardt, Observations of energetic ions and electrons in Saturn's magnetosphere, Science, $207,421,1980$.

Van Allen, J. A., Absorption of energetic protons by Saturn's ring G, J. Geophys. Res., 88, 6911, 1983.

Van Allen, J. A., M. F. Thomsen, B. A. Randall, R. L. Rairden, and C. L. Grosskreutz, Saturn's magnetosphere, rings, and inner satellites, Science, 207, 415, 1980a.

Van Allen, J. A., M. F. Thomsen, and B. A. Randall, The energetic charged particle absorption signature of Mimas, J. Geophys. Res. $85,5709,1980 b$.

Vogt, R. E., D. L. Chenette, A. C. Cummings, T. L. Garrard, E. C. Stone, A. W. Schardt, J. H. Trainor, N. Lal, and F. B. McDonald, Energetic charged particles in Saturn's magnetosphere: Voyager 2 results, Science, 215, 577, 1982.

(Received February 2, 1983;

revised April, 21, 1983;

accepted April 22, 1983.) 